\title{
Comportamento germinativo de duas espécies de canga ferrífera: Baccharis retusa DC. (Asteraceae) e Tibouchina multiflora Cogn. (Melastomataceae)
}

\author{
Letícia Couto Garcia ${ }^{1,2}$, Fernanda de Vasconcellos Barros ${ }^{1,2}$ e José Pires Lemos Filho ${ }^{1,3}$
}

Recebido em 17/09/2004. Aceito em 13/10/2005

\begin{abstract}
RESUMO -(Comportamento germinativo de duas espécies de canga ferrífera: Baccharis retusa DC. (Asteraceae) e Tibouchina multiflora Cogn. (Melastomataceae)). O objetivo desse estudo foi avaliar o comportamento germinativo de Bacccharis retusa e Tibouchina multiflora, espécies que ocorrem na vegetação de canga no Quadrilátero Ferrífero de Minas Gerais. Diásporos coletados no município de Barão de Cocais foram colocados para germinar nas temperaturas de $15,20,25$ e $30{ }^{\circ} \mathrm{C}$ na presença de luz contínua ou no escuro. As sementes mantidas inicialmente no escuro foram posteriormente transferidas para a presença de luz, mantendo-se as mesmas temperaturas. Os diásporos das duas espécies apresentaram comportamento fotoblástico positivo, com germinação inexpressiva no escuro em todas as temperaturas testadas. Na presença de luz contínua as sementes de T. multiflora não apresentaram diferenças na porcentagem final de germinação em todas as temperaturas testadas, mas B. retusa apresentou menor porcentagem final de germinação a $30{ }^{\circ} \mathrm{C}$. Os diásporos das duas espécies mantidos inicialmente no escuro, germinaram rapidamente após serem transferidos para luz branca contínua. Entretanto, não foi verificado efeito significativo do tempo de permanência no escuro nos valores finais de porcentagem de germinação alcançados a cada temperatura. Esses resultados sugerem que essas duas espécies de canga apresentam potencial para a formação de banco de sementes no solo.
\end{abstract}

Palavras-chave: banco de sementes, campo rupestre, fotoblastismo, luz, temperatura

\begin{abstract}
Germinative behavior of two species from "canga ferrífera": Baccharis retusa DC. (Asteraceae) and Tibouchina multiflora Cogn. (Melastomataceae)). The aim of this study was to evaluate the germinative behavior of Bacccharis retusa and Tibouchina multiflora, species that occur in the "canga" vegetation of the Quadrilátero Ferrífero of Minas Gerais. Diaspores collected in the county Barão de Cocais were placed to germinate under the temperatures of $15,20,25$ and $30{ }^{\circ} \mathrm{C}$ at continuous light or in darkness condition. The diaspores initially remained under darkness were later transferred to light presence maintaining the same temperatures. Diaspores of both species presented a positive photoblastic behavior, with inexpressive germination under darkness in all tested temperatures. In presence of continuous light, seeds of T. multiflora showed no difference in the percentage of germination in all temperatures, while $B$. retusa showed lower germination at $30{ }^{\circ} \mathrm{C}$. Diaspores initially maintained under darkness germinated rapidly after transferred to continuous light. However, no indication of significant effect of the dark period was observed in values of the final germination percentage in each temperature tested. These results suggest that these two "canga" species may have potential to constitute the soil seed bank
\end{abstract}

Key words: seed bank, rupestrian field, photoblastism, light, temperature

\section{Introdução}

A germinação das sementes é uma das fases mais críticas do ciclo de vida das plantas e determina tanto a distribuição das espécies como sua abundância nas comunidades vegetais. Os estudos sobre germinação permitem a compreensão dos fatores limitantes para o estabelecimento, sobrevivência e regeneração das espécies (Black \& El Hadi 1992; Vázquez-Yanes \& Orozco-Segovia 1993).
O estabelecimento de plântulas nos diferentes habitats é determinado pelas características fisiológicas e bioquímicas das sementes durante o processo de germinação (Bewley \& Black 1994), que ocorre quando as condições externas são favoráveis. Entre os fatores ambientais, a temperatura e a luz são considerados os mais limitantes para o processo germinativo (Baskin \& Baskin 1998). A temperatura influi tanto na porcentagem como na velocidade de germinação por afetar a embebição e os processos

\footnotetext{
1 Universidade Federal de Minas Gerais, Departamento de Botânica, ICB, Laboratório de Fisiologia Vegetal, CEP 31270-110, Belo Horizonte, MG, Brasil

2 Pós-graduação em Ecologia, Conservação e Manejo da UFMG

3 Autor para correspondência: lemos@icb.ufmg.br
} 
metabólicos (Castro \& Hilhorst 2004), enquanto que a percepção da luz ambiental, mediada pelo fitocromo (Lindig-Cisneiros \& Zedler 2001), é necessária para a germinação de muitas espécies em habitats ensolarados (Baskin \& Baskin 1971). Essas espécies, normalmente, apresentam sementes pequenas com uma reserva limitada de nutrientes, que será exaurida antes que possa se estabelecer a autotrofia, limitando, portanto a sobrevivência quando enterradas em maior profundidade no solo (Bewley \& Black 1994).

Baccharis retusa DC. (Asteraceae) e Tibouchina multiflora Cogn. (Melastomataceae) são duas espécies arbustivas encontradas freqüentemente em áreas de canga no Quadrilátero Ferrífero de Minas Gerais, onde a atividade mineradora é intensa. Essa região é caracterizada por vegetação de campo rupestre sobre rocha ferruginosa cenozóica composta por fragmentos de hematita cimentados por limonita, conhecida como canga (Rizzini 1979). Estudos sobre a germinação das sementes de espécies que ocorrem nas áreas de canga ferrífera são escassos e poderiam contribuir para a conservação e manejo, uma vez que muitas dessas espécies se encontram sujeitas à grande pressão antrópica pela urbanização e, principalmente pela atividade mineradora.

Já existem alguns estudos sobre germinação de espécies do gênero Baccharis, $B$. dracunculifolia (Gomes \& Fernandes 2002) e B. trimera (Ferreira et al. 2001) e do gênero Tibouchina, T. benthamiana Cogn., T. moricandiana (DC.) Baill., T. grandiflora (Andrade 1995) e T. pulchra, T. granulosa (Zaia \& Takaki 1998); porém, na literatura ainda não se encontram dados sobre germinação de $B$. retrusa e de T. multiflora. Considerando-se as características dos sítios de ocorrência das duas espécies, a canga ferrífera, um ambiente aberto e ensolarado e o pequeno tamanho das sementes, é esperado um comportamento fotoblástico positivo, e que os diásporos apresentem respostas germinativas também similares em relação à temperatura. Com base nessas premissas, o presente estudo visou determinar o comportamento germinativo de aquênios de $B$. retusa e de sementes de T. multiflora na presença e ausência de luz branca contínua sob diferentes temperaturas.

\section{Material e métodos}

Diásporos de três indivíduos adultos de Baccharis retusa DC. e Tibouchina multiflora Cogn. foram coletados em uma área próxima ao município de Barão

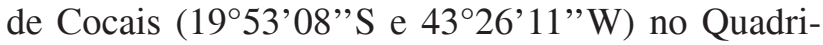

látero Ferrífero de Minas Gerais a uma altitude de $1.120 \mathrm{~m}$. A vegetação local é um campo rupestre em canga couraçada, com as espécies ocorrendo em substratos ricos em minério de ferro (Vicent et al. 2002). Nessa região, a estação chuvosa ocorre de novembro a março e a estação seca, de abril a outubro. Outra característica ambiental de áreas de canga é a grande amplitude térmica diária devido ao intenso aquecimento da rocha durante o dia e seu resfriamento noturno (Vicent et al. 2002).

No laboratório foram descartados os diásporos danificados a fim de homogeneizar o lote para os experimentos de germinação. Cinqüenta aquênios de $B$. retusa e o mesmo número de sementes de T. multiflora foram medidos no seu maior comprimento e largura com auxílio de um paquímetro digital. Os diásporos foram colocados para germinar em placas gerbox forradas com folha dupla de papel filtro embebido com solução de nistatina a 1\% (Gomes \& Fernandes 1994; Ranieri et al. 2003). As placas foram mantidas em câmara de germinação nas temperaturas constantes de $15,20,25$ e $30{ }^{\circ} \mathrm{C}$, no escuro ou sob luz branca contínua fornecendo cerca de $30 \mu \mathrm{mol} . \mathrm{m}^{-2} . \mathrm{s}^{-1}$ de radiação fotossintéticamente ativa, determinada com sensor quântico LI-COR. Para cada tratamento foram utilizadas quatro repetições com 25 diásporos cada. Foram usadas placas pintadas de preto, colocadas em sacos plásticos pretos para a obtenção de escuro contínuo. As contagens foram realizadas diariamente e a germinação nas placas mantidas no escuro foi verificada sob luz verde de segurança. Foi adotado como critério de germinação a protusão radicular (Lucas \& Arrigoni 1992). A duração do experimento foi de 57 dias para $B$. retusa e 30 dias para T. multiflora, sendo que após 22 dias de permanência no escuro os diásporos foram transferidos para luz contínua sem alteração da temperatura e permaneceram nessa condição por mais 35 dias (B. retusa) e 14 dias (T. multiflora). Por ocasião do encerramento da avaliação da germinação, a maior parte das sementes remanescentes não germinadas apresentava visíveis sinais de deterioração com ataque de microorganismos.

Os resultados foram analisados a partir de cálculos de porcentagem final $(\mathrm{G})$ e o tempo médio de germinação $(\overline{\mathrm{t}})$ segundo a fórmula proposta por Labouriau (1983): $\mathrm{G}=\Sigma \mathrm{n}_{\mathrm{i}} / \mathrm{N} \times 100$, onde $\mathrm{n}_{\mathrm{i}}$ é o número de sementes germinadas e $\mathrm{N}$ é o número de sementes colocadas em cada placa; $\overline{\mathrm{t}}=\Sigma\left(\mathrm{n}_{\mathrm{i}} \times \mathrm{t}_{\mathrm{i}}\right) / \Sigma \mathrm{n}_{\mathrm{i}}$, onde ni é o número de diásporos germinados em cada dia e $t_{i}$ é o dia em que estes germinaram. Os valores 
obtidos foram verificados quanto a sua distribuição normal pelo teste Kolmogorov-Smirnov, utilizando-se o programa STATISTICA 6.0. Essa análise apontou que somente os dados de tempo médio de germinação de T. multiflora não alcançaram normalidade e dessa forma, foram transformados em log $(\mathrm{X}+1)$ (Santana \& Ranal 2004). Em seguida, os resultados foram submetidos à análise de variância e as médias comparadas pelo teste de Tukey $(\mathrm{p}<0,05)$, empregando-se o software VARPC. O cálculo de germinabilidade considerou todas as sementes germinadas do início ao término dos experimentos, enquanto que o tempo médio das sementes que inicialmente foram mantidas no escuro, foi computado a partir da transferência para a luz, sendo excluído o tempo em que não houve germinação, durante o período no escuro.

\section{Resultados}

As medidas de comprimento e largura dos diásporos, das duas espécies estudadas, foram respectivamente $2,26( \pm 0,23) \mathrm{mm}$ e $0,77( \pm 0,11) \mathrm{mm}$ em $B$. retusa e $0,52( \pm 0,09) \mathrm{mm}$ e $0,37( \pm 0,08) \mathrm{mm}$ para T. multiflora. Conforme o esperado para sementes de ambientes ensolarados e de pequenas dimensões, foi verificado um comportamento fotoblástico positivo para ambas as espécies, porém respostas diferenciais de germinação em relação à temperatura (Fig. 1 e 2). Na presença contínua de luz não foram verificadas diferenças significativas nos valores finais da porcentagem de germinação das sementes de T. multiflora, mas os diásporos de B. retusa apresentaram menor porcentagem a $30^{\circ} \mathrm{C}$. Quando foram transferidos para a luz, os diásporos que estavam no escuro começaram a germinar após quatro dias em T. multiflora e após nove dias em $B$. retusa. Nas duas espécies, não foi verificado efeito significativo do tempo de permanência no escuro nos valores finais de porcentagem de germinação alcançado em cada temperatura. Os valores máximos de porcentagem de germinação foram $73 \%$ para T. multiflora e $93 \%$ para B. retusa.

O tempo médio de germinação $(\overline{\mathrm{t}})$ é um indicativo da velocidade do processo germinativo e pode ser usado conforme o proposto por Ferreira et al. (2001), para classificar as sementes em três categorias; com geminação rápida $(<5$ dias $)$, germinação com valores intermediários $(5<\overline{\mathrm{t}}<10$ dias $)$ e por último aquelas com germinação lenta ( $>10$ dias). Considerando essa classificação, conforme pode ser verificado na Fig. 3, as sementes de $T$. multiflora germinadas sob luz branca contínua apresentaram, em geral, valores intermediários de tempo médio de germinação, sendo que os menores valores de tempo médio de germinação foram registrados a 25 e $30{ }^{\circ} \mathrm{C}$. Na temperatura de $15{ }^{\circ} \mathrm{C}$ a germinação foi lenta, diferindo significativamente dos valores obtidos para as outras temperaturas. Porém, os diásporos de $B$. retusa, na presença de luz, apresentaram germinação lenta sob todas as temperaturas testadas (Fig. 4), com o tempo médio a $15{ }^{\circ} \mathrm{C}$ menor que a $30{ }^{\circ} \mathrm{C}$.

Quando transferidos do escuro para a presença de luz branca contínua, as sementes de T. multiflora apresentaram tempo médio de germinação menor a 25 e $30{ }^{\circ} \mathrm{C}$ em relação às temperaturas mais baixas. Em $B$. retusa o menor tempo médio foi alcançado na temperatura de $20{ }^{\circ} \mathrm{C}$ (Fig. 4). Diásporos mantidos

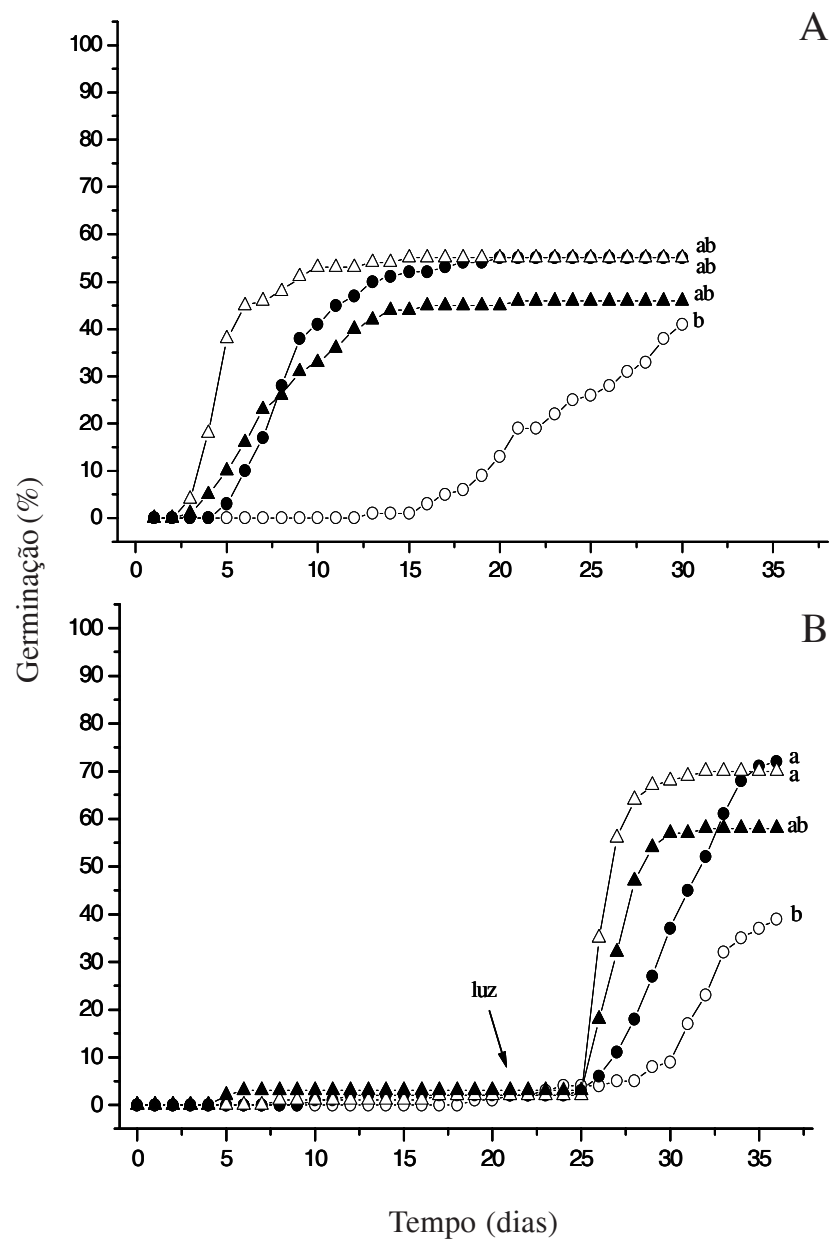

Figura 1. Porcentagem acumulada de germinação de sementes de Tibouchina multiflora Cogn. submetidas a diferentes temperaturas sob luz branca contínua (A), sob escuro e depois transferidas para luz branca contínua (B). Porcentagens seguidas de mesma letra não diferem entre si pelo teste de Tukey a $5 \%$ de probabilidade. $-\mathrm{O}-=15^{\circ} \mathrm{C} ;-\bullet-=20^{\circ} \mathrm{C} ;-\triangle-=25^{\circ} \mathrm{C} ;-\boldsymbol{\Delta}-=30{ }^{\circ} \mathrm{C}$. 
inicialmente no escuro e depois transferidos para luz, quando comparados com aqueles obtidos nos tratamentos em luz branca contínua, apresentaram menores valores de tempo médio de germinação (Fig. 3, 4). Em T. multiflora o processo de germinação foi acelerado significativamente a 15 e $30^{\circ} \mathrm{C}$, enquanto em $B$. retusa isso ocorreu a 20,25 e $30^{\circ} \mathrm{C}$.

\section{Discussão}

Os resultados obtidos neste estudo para $T$. multiflora e $B$. retusa indicam a presença de um comportamento fotoblástico positivo para estas espécies, que apresentaram uma germinação inexpressiva no escuro em todas as temperaturas testadas. Tal comportamento também foi observado em estudos realizados com outras espécies de

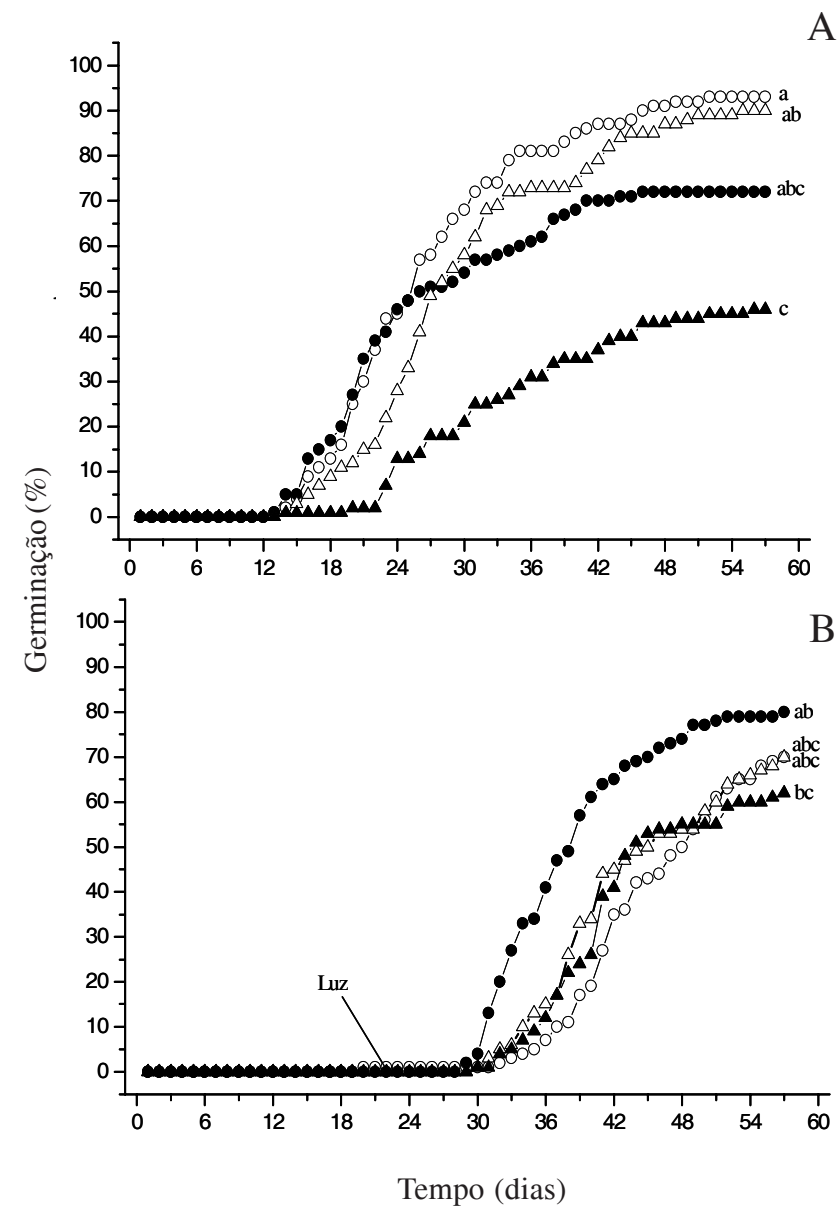

Figura 2. Porcentagem acumulada de germinação de aquênios de Baccharis retusa DC. submetidos a diferentes temperaturas sob luz branca contínua (A), sob escuro e depois transferidos para luz branca contínua (B). Porcentagens seguidas de mesma letra não diferem entre si pelo teste de Tukey a $5 \%$ de significância. $-\mathrm{O}-=15^{\circ} \mathrm{C} ;-\bullet-=20^{\circ} \mathrm{C} ;-\triangle-=25^{\circ} \mathrm{C} ;-\boldsymbol{\Delta}-=30^{\circ} \mathrm{C}$.
Tibouchina, T. grandiflora, T. benthamiana, T. moricandiana (Andrade 1995) e sementes de T. pulchra e $T$. granulosa avaliadas na temperatura de $25^{\circ} \mathrm{C}$ (Zaia \& Takaki 1998). No gênero Baccharis, a intensidade do fotoblastismo pode variar entre as espécies. B. trimera foi classificada como fotoblástica positiva, pois o percentual de germinação na luz foi mais que o dobro do que o observado no escuro

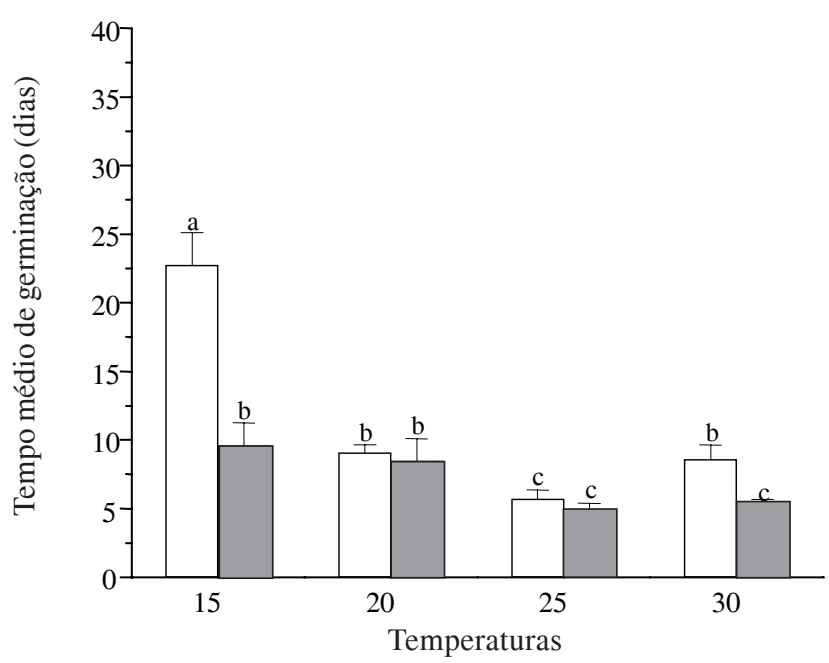

Figura 3. Tempo médio de germinação de sementes de Tibouchina multiflora Cogn. submetidas a diferentes temperaturas sob irradiância durante 30 dias (barra vazia) e transferidas para luz branca contínua durante 14 dias (barra cheia). Valores seguidos de mesma letra não diferem entre si pelo teste de Tukey a $5 \%$ de probabilidade. $\square=$ Luz (30 dias); $\square=$ Transferência para a luz (14 dias).

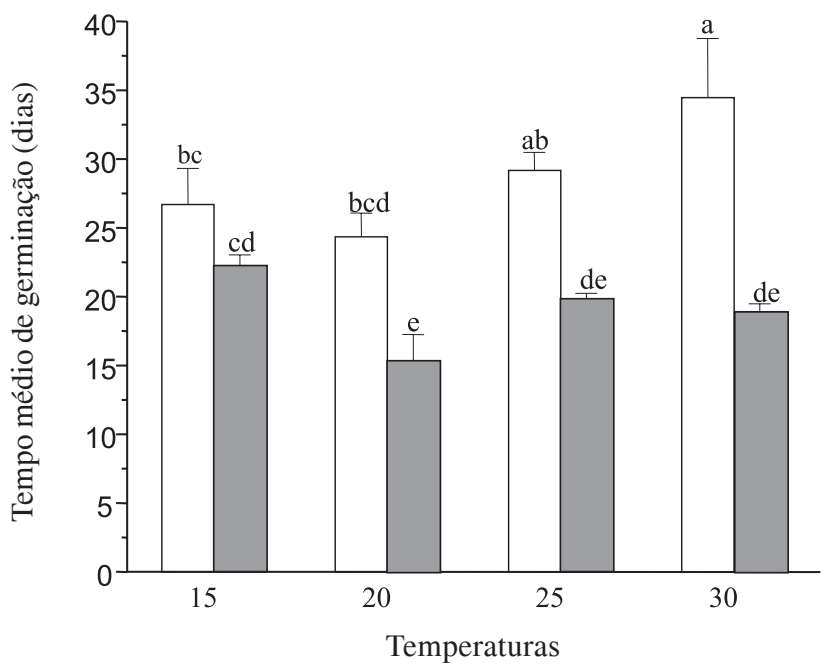

Figura 4. Tempo médio de germinação de aquênios de Baccharis retusa DC. submetidas a diferentes temperaturas sob irradiância durante 57 dias (barra vazia) e transferidas para luz branca contínua durante 35 dias (barra cheia). Valores seguidos de mesma letra não diferem entre si pelo teste de Tukey a $5 \%$ de probabilidade. $\square=\operatorname{Luz}$ (57 dias); $\square=$ Transferência para a luz (35 dias). 
(Ferreira et al. 2001). Já os diásporos de $B$. dracunculifolia germinaram bem na presença de luz e no escuro a $15^{\circ} \mathrm{C}$ e apresentaram comportamento fotoblastico positivo a 20,25 e $30{ }^{\circ} \mathrm{C}$ (Gomes \& Fernandes 2002). De acordo com Takaki (2001) o fotoblastismo está relacionado às formas do fitocromo que controlam a geminação, já que todas a sementes possuem esse pigmento e este seria responsável pela percepção das condições de luminosidade do ambiente.

Frequientemente as condições ambientais exigidas para a germinação estão relacionadas com as condições ecológicas predominantes no habitat da planta ou da semente. Normalmente, as temperaturas em que ocorrem as maiores taxas germinativas correspondem aos valores registrados nas épocas e microambientes mais propícios para o recrutamento de plântulas (Baskin \& Baskin 1992). Espécies euritérmicas quanto à germinação, possuem uma habilidade competitiva maior que as estenotérmicas (Baskin \& Baskin 1998). Dessa forma, os altos valores das porcentagens finais de germinação observados nos diferentes tratamentos de temperatura para T. multiflora, sob luz branca contínua, podem contribuir significativamente para o sucesso no recrutamento dessa espécie da mesma forma como o descrito para espécies de Vellozia de campo rupestre (Garcia \& Diniz 2003). O comportamento euritérmico em T. multiflora pode estar relacionado à ampla variação na temperatura ao longo do período de dispersão dessa espécie, que vai de abril a novembro, conforme observações fenológicas realizadas em campo. Porém, deve ser salientado que o tempo médio de germinação a $15^{\circ} \mathrm{C}$ foi maior que o observado a 20 , 25 e $30^{\circ} \mathrm{C}$ evidenciando o efeito limitante das baixas temperaturas na embebição e na atividade metabólica das sementes (Castro \& Hilhorst 2004). Maiores velocidades de germinação em temperaturas mais elevadas também foram observadas por Andrade (1995) a 25 e $30{ }^{\circ} \mathrm{C}$ para sementes de três espécies de Tibouchina.

Na presença de luz contínua, $B$. retusa apresentou um comportamento diverso do constatado para T. multiflora, com menor porcentagem final de germinação e maior tempo médio a $30^{\circ} \mathrm{C}$. Isso condiz com sua rápida dispersão no mês de julho, época em que as temperaturas locais são mais baixas. Uma menor velocidade de germinação verificada a $30{ }^{\circ} \mathrm{C}$, em comparação às menores temperaturas, também foi observada em um estudo realizado com B. dracunculifolia (Gomes \& Fernandes 2002), ainda que não tenham constatado diferenças significativas na porcentagem final de germinação entre as temperaturas de 15,20 e $30^{\circ} \mathrm{C}$. Ferreira et al. (2001) verificaram que aquênios de $B$. trimera, ao contrário do observado no presente estudo com $B$. retusa, apresentaram baixos valores de porcentagem de germinação (menores que 35\%) e germinação rápida, sem diferenças entre as temperaturas estudadas, de 20 a $30^{\circ} \mathrm{C}$. O longo período de germinação observado para $B$. retusa pode estar relacionado com a necessidade de um ambiente mais estável e úmido para o estabelecimento das plântulas, conforme as considerações apresentadas por Ranieri et al. (2003) para Lavosiera cordata (Melastomataceae).

Os diásporos, de ambas espécies estudadas, submetidos ao escuro com posterior transferência para a luz, germinaram rapidamente, como pode ser observado pelo aumento da inclinação das curvas (Fig. 1,2) e pelos menores valores de tempo médio a 15 e $30{ }^{\circ} \mathrm{C}$, para T. multiflora, e a 20,25 e $30{ }^{\circ} \mathrm{C}$ para B. retusa (Fig. 3, 4). Essa resposta é similar à descrita para duas espécies de Bromeliaceae, sugerindo a capacidade de formação de um banco de sementes temporário no solo (Pinheiro \& Borghetti 2003). Desse modo, as sementes enterradas e embebidas manifestarão germinação mais rápida quando expostas à luz, já que o processo de embebição inicia a respiração da semente aumentando a quantidade de ATP que será utilizado para os processos de iniciação do crescimento do embrião (Castro \& Hilhorst 2004). Os diásporos de $B$. retusa mantidos inicialmente no escuro apresentaram um tempo médio significativamente menor a $20{ }^{\circ} \mathrm{C}$, o que poderia indicar um bom desempenho germinativo nessa faixa de temperatura após um período de embebição. Já em T. multiflora, as temperaturas mais elevadas $\left(25\right.$ e $\left.30{ }^{\circ} \mathrm{C}\right)$ é que exerceriam influência positiva na germinação a partir do banco de sementes. Comparando-se o comportamento germinativo de $B$. retusa e T. multiflora, foi verificado, conforme o esperado, a ocorrência de comportamento fotoblástico positivo, comumente encontrado para espécies com sementes pequenas (Bewley \& Black 1994). Entretanto, apesar da ocorrência em simpatria, os diásporos das duas espécies estudadas apresentam comportamento distinto em relação à temperatura, fato possivelmente relacionado com o período de dispersão dos diásporos.

\section{Agradecimentos}

Os autores agradecem a Cia Vale do Rio Doce, pelo apoio financeiro; ao CNPq, pela bolsa de Iniciação Científica. José Pires Lemos Filho agradece ao CNPq, pela bolsa de pesquisa. 


\section{Referências bibliográficas}

Andrade, A.C.S. 1995. Efeito da luz e da temperatura na germinação de Leandra breviflora Cong., Tibouchina benthamiana Cong., Tibouchina grandiflora Cong. e Tibouchina moricandiana (DC.) Baill. (Melastomataceae). Revista Brasileira de Sementes 17: 29-35.

Baskin, J.M. \& Baskin, C.C. 1971. The possible ecological significance of the light requirement for germination of Cyperus inflexus. Bulletin of the Torrey Botanical Club 98: 25-33.

Baskin, J.M. \& Baskin, C.C. 1992. Role of temperature and light in the germination ecology of buried seeds of weedy species of disturbed forests. I. Lobelia inflata. Canadian Journal of Botany 70: 589-592.

Baskin, J.M. \& Baskin, C.C. 1998. Seeds, ecology, biogeography, and evolution of dormancy and germination. New York, Academic Press.

Bewley, J.D. \& Black, M. 1994. Seeds: Physiology of development and germination. New York, Plenum Press.

Black, A.R. \& El Hadi, F.M.1992. Presouring treatments of Acacia senegal seed germination and growth. Tropical Agricultural 69: 15-20

Castro, R.D. \& Hilhorst, H.W.M. 2004. Embebição e reativação do metabolismo. Pp.149-162. In: A.G. Ferreira \& F. Borghetti (eds.). Germinação: do básico ao aplicado. Porto Alegre, Artmed.

Ferreira, A.G.; Cassol, B.; Rosa, S.G.T.; Silveira, T.S.; Stival, A.L. \& Silva, A.A. 2001. Germinação de sementes de Asteraceae nativas no Rio Grande do Sul, Brasil. Acta Botanica Brasilica 15: 231-242.

Garcia, Q.S. \& Diniz, I.S.S. 2003. Comportamento germinativo de três espécies de Vellozia da Serra do Cipó, MG. Acta Botanica Brasilica 17(4): 487-494.

Gomes, V. \& Fernandes, G.W. 2002. Germinação de aquênios de Baccharis dracunculifolia DC. (Asteraceae). Acta Botanica Brasilica 16: 421-427.
Labouriau, L.G. 1983. A germinação das sementes. Washington, The General Secretariat Of the Organization of American States.

Lindig-Cisneiros, R. \& Zedler, J. 2001. Effects of light on seed germination in Aphalaris arundinaceae L. (reed canary grass) Plant Ecology 155: 75-78.

Lucas, N.M.C. \& Arrigoni, M.F. 1992. Germinação de sementes de Canavalia rosea (Sw.) DC. (Fabaceae). Revista Brasileira de Botânica 15: 105-112.

Pinheiro, F. \& Borghetti, F. 2003. Light and requirements for germination of seeds of Aechmea nudicaulis (L.) Griesebach and Streptocalyx floribundus (Martius ex Schultes F.) Mez (Bromeliaceae). Acta Botanica Brasilica 17: 27-35.

Ranieri, B.D.; Lana, T.C.; Negreiros, D.; Araújo, L.M.; Fernandes, G.W. 2003. Germinação de sementes de Lavosiera cordata Cogn. e Lavosiera francavillana (Melastomataceae), espécies simpátricas da Serra do Cipó, Brasil. Acta Botanica Brasilica 17: 523-530.

Rizzini, C.T. 1979. Tratado de fitogeografia do Brasil. $2^{\text {a }} \mathrm{ed.}$ São Paulo, Hucitec/Editora da Universidade de São Paulo.

Santana, D.G. \& Ranal, M.A. 2004. Análise da germinação: Um enfoque estatístico. Brasília, Editora UnB.

Takaki, M. 2001. Comunication - New proposal of classification of seeds based on forms of phytocrome instead of photoblastism. Revista Brasileira de Fisiologia Vegetal 13(1): 104-108.

Vázquez-Yanes, C. \& Orozco-Segovia, A. 1993. Patterns of seed longevity and germination in the tropical rainforest. Annual Review of Ecology and Systematics 24: 69-87.

Vicent, R.C.; Jacobi, C.M. \& Antonini, Y. 2002. Diversidade na adversidade. Ciência Hoje 31(185): 64-67.

Zaia, J.E. \& Takaki, M. 1998. Estudo da germinação de sementes de espécies arbóreas pioneiras: Tibouchina pulchra Cong. e Tibouchina granulosa Cong. (Melastomataceae). Acta Botanica Brasilica 12: 221-229. 